\title{
Parameter Sensitivity Analysis of Two Low-Cost Sensorless Induction Motor Drives
}

\author{
S. Bolognani ${ }^{*}$, A.Faggion*, L. Peretti** and M. Zigliotto** \\ ${ }^{*}$ University of Padova, Dept. of Electrical Engineering, Via Gradenigo 6/A, 35131 Padova, Italy \\ ${ }^{* *}$ University of Padova, Dept. of Technique and Management of Industrial Systems, Stradella San Nicola 3, 36100 \\ Vicenza, Italy
}

\begin{abstract}
The paper presents a detailed sensitivity analysis comparison between two existing low-cost sensorless solutions for induction motor drives. The first solution is a $V$-Type approach, in which the inverter voltage references are calculated from the machine equations instead of obtained as the output of PI current regulators. The scheme is obtained from a standard field oriented control drive by successive approximations. The second solution is an openloop speed estimator, based on the machine equations only, used in conjunction with a field oriented control drive with speed and current closed loops. An analytical sensitivity analysis has been carried out first, looking for the behaviour of the two schemes in presence of single motor parameter mismatch. Both schemes have been implemented in laboratory prototypes, and their performances have been tested in different conditions. The results of the comparison are discussed in details. A side value is the generality of the proposed analysis method, which can be applied as a standard comparison tool for control schemes evaluation.
\end{abstract}

\section{INTRODUCTION}

In last decades, many sensorless schemes with very different approaches have been proposed for induction motor (IM) drives. A good classification can be found in [1]. A rather numerous family is composed by closed-loop algorithms, where the speed estimation is obtained by means of a motor model and either a linear or non-linear controller. Examples are Luenberger observers [2], Kalman filters [3] or model reference adaptive systems (MRAS) [4].

Another category is composed by open-loop algorithms, which are interesting for their low-cost profile suitable for some industrial applications [5], [6]. In openloop approaches, the speed is estimated by means of the machine equations only, without the need of a controller. Very often, open loop controls are tuning-free and the related digital computational effort is lower with respect to a closed-loop solution. Major disadvantages are the parameter variations sensitivity and the implementation of integral and differential blocks, which need proper countermeasures to avoid the well-known output drift problem.

After a first research step, aimed to find different openloop schemes for IMs, the attention is now put on their parameter variations sensitivity, which is a key element for a correct comparison between the algorithms. In this paper, a comparison between two different open-loop sensorless topologies for IM drives is presented, focusing on parameter sensitivity analysis. The first algorithm is an approximation of a standard field oriented control (FOC) drive. It can be considered as an enhanced scalar solution with respect to a classical $\mathrm{V} / \mathrm{Hz}$ algorithm, with very low computational requirements. The second solution is an open-loop speed estimator (OLSE), based on the machine equations only. The OLSE is used in combination with a standard FOC drive for IM to provide a fully sensorless vector drive. On one hand, the OLSE solution cannot be considered as a closed-loop estimator, since it just exploits machine parameters, current and voltages to estimate the speed. On the other, it cannot be considered as a pure open-loop solution, since it is used in combination with a standard FOC drive, simply replacing the feedback signal from the speed sensor.

First, a theoretical sensitivity analysis is carried out, studying the behaviour of the two schemes in presence of a motor parameter mismatch. Starting from the mathematical description of the two solutions, the sensitivity functions have been calculated and compared for each motor parameter, as function of the exciting frequency and the load torque.

Then, the two schemes have been implemented on a fast control prototyping test bed and an extended batch of measures has been carried out for validation purposes.

As detailed in the next sections, the approach used to calculate the analytical sensitivity functions was not the same for both the sensorless algorithms, due to their slightly different control concepts.

As a value added, the presented method of analysis for parameter sensitivity is of general validity, and it can be applied as a standard tool during schemes evaluation, which is an emerging request by the industrial partners. The procedure is also potentially useful for the on-line acknowledgement and compensation of parameters mismatch.

\section{V-TYPE DRIVE}

The first sensorless algorithm is a V-Type drive, an open-loop solution where the voltage references for the inverter are directly calculated instead of obtained as the output of current regulators, which are not present any more. The algorithm is derived from the conventional FOC equations by successive approximations, as reported in [7], with the recent evolutions described in [8].

Motor dynamics equations are written in a generic frame rotating at a speed $\omega_{g}$, as reported hereafter. It is worth to note that the IM is modelled with its equivalent four-parameters electrical circuit, which comprises the 
stator resistance $R_{s}$, the rotor resistance referred to the stator $R_{s}$, the transient inductance $L_{t}$ and the magnetising inductance $L_{\varphi}$.

$$
\frac{d}{d t}\left[\begin{array}{l}
i_{s} \\
\lambda_{s r}
\end{array}\right]=\left[\begin{array}{ll}
A_{11} & A_{12} \\
A_{21} & A_{22}
\end{array}\right]\left[\begin{array}{l}
i_{s} \\
\lambda_{s r}
\end{array}\right]+\left[\begin{array}{l}
B_{1} \\
0
\end{array}\right] u_{s}
$$

Vectors and matrices assume the following form:

$$
\begin{aligned}
& i_{s}=\left[\begin{array}{ll}
i_{s d} & i_{s q}
\end{array}\right]^{T} \quad \text { stator currents } \\
& \lambda_{s r}=\left[\begin{array}{ll}
\lambda_{s r d} & \lambda_{s r q}
\end{array}\right]^{T} \quad \text { rotor fluxes } \\
& u_{s}=\left[\begin{array}{ll}
u_{s d} & u_{s q}
\end{array}\right]^{T} \quad \text { stator voltages } \\
& A_{11}=-\frac{R_{s}+R_{s r}}{L t} I-\omega_{g} J \\
& A_{12}=\frac{R_{s r}}{L_{t} L_{\varphi}} I-\omega_{m e} \frac{1}{L_{t}} J \\
& A_{21}=R_{s r} I \\
& A_{22}=-\frac{R_{s r}}{L_{\varphi}} I-\left(\omega_{g}-\omega_{m e}\right) J \\
& B_{1}=\frac{1}{L_{t}} I \\
& I=\left[\begin{array}{ll}
1 & 0 \\
0 & 1
\end{array}\right] \\
& J=\left[\begin{array}{cc}
0 & -1 \\
1 & 0
\end{array}\right]
\end{aligned}
$$

In addition to (1), a complete description of the system is obtained with the following equation, which relates the output torque with the mechanical load:

$$
\frac{d \omega_{m e}}{d t}=\frac{3}{2} \frac{p^{2}}{J_{m}}\left(\lambda_{s r d} i_{s q}-\lambda_{s r q} i_{s d}\right)-\frac{B_{m}}{J_{m}} \omega_{m e}-\frac{p}{J_{m}} \tau_{L}
$$

The voltage references, conventionally produced by PI current controllers, are obtained from (1) and (2) by replacing the voltages $u_{d}$ and $u_{q}$ with the IM voltage balance equations at steady-state [7]:

$$
\begin{aligned}
& u_{s d}=R_{s}^{*} i_{s d}-\omega_{g} L_{t}^{*} i_{s q} \\
& u_{s q}=R_{s}^{*} i_{s q}+\omega_{g}\left(L_{t}^{*} i_{s d}+\lambda_{s r d}^{*}\right)
\end{aligned}
$$

Control model parameters in (3) are denoted by asterisks superscripts, and they do not necessarily coincide with the actual values. $\lambda_{s r d}^{*}$ is the reference value for the $\mathrm{d}$-axis rotor flux linkage referred to the stator.

If $\omega_{g}$ is assumed to be the rotor flux linkage vector speed [7], a conventional FOC drive approximation is obtained. The speed $\omega_{g}$ is obtained as the sum of the slip speed and the mechanical rotor speed, the latter being approximated by the rotor speed reference in the sensorless approach:

$$
\omega_{g}=\omega_{\lambda_{r}} \approx \omega_{m e}^{*}+\omega_{s l}^{*}=\omega_{m e}^{*}+\frac{R_{s r}^{*}}{L_{\varphi}^{*}} \frac{i_{s q}}{i_{s d}}
$$

The slip speed is obtained by means of the current measurements as proposed in [8], instead of using the flux reference signal on the $\mathrm{d}$ axis as used in [7]. Both the cited works have also demonstrated that the system is unstable if the $i_{s d}$ and $i_{s q}$ are directly used in (3) and (4). Two filtered values must be used instead, as shown hereafter:

$$
i_{D, Q}=\frac{1}{1+s T_{f}} i_{s d, s q}
$$

The complete scheme of the V-type solution is reported in Figure 1.

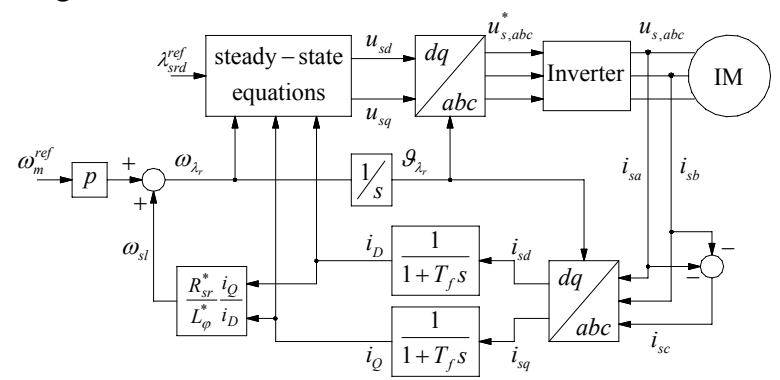

Figure 1. Sensorless V-Type block schematic.

It is worth to note that the system, as a whole, can be expressed in a matrix notation as:

$$
\frac{d x}{d t}=A(p, x)
$$

where $x=\left[\begin{array}{lllllll}i_{s d} & i_{s q} & l_{s r d} & l_{s r q} & i_{D} & i_{Q} & \omega_{m e}\end{array}\right]^{T}$ and $p=\left[R_{s}{ }^{*} R_{s r}{ }^{*} L_{t}{ }^{*}\right.$ $\left.L_{\varphi}{ }^{*}\right]^{T}$. This notation will be useful for the sensibility analysis performed in Sec. IV.

\section{DRIVE BASED ON OPEN LOOP SPEED ESTIMATION (OLSE)}

The second sensorless algorithm is described in [9]. It is also based on the four-parameter equivalent electrical circuit of the IM, and it can be obtained from the vector equation that describes the motor dynamics:

$$
\frac{d \lambda_{s r}^{s}}{d t}=\left(j \omega_{m e}-\frac{R_{s r}}{L_{\varphi}}\right) \lambda_{s r}^{s}+R_{s r} i_{s}^{s}
$$

where $i_{s}{ }^{s}$ is the stator current vector and $\lambda_{\mathrm{sr}}{ }^{\mathrm{s}}$ is the rotor flux linkage vector referred to the stator. The equation refers to an the $\alpha \beta$ reference frame, fixed to the stator coordinates. Solving (7) for $\omega_{m e}$, multiplying numerator and denominator by $\lambda_{\mathrm{sr}}{ }^{\mathrm{s}^{*}}$ (complex conjugate of $\lambda_{\mathrm{sr}}^{\mathrm{s}}$ ) and taking only the real part (since $\omega_{m e}$ is real), one obtains:

$\hat{\omega}_{m e}=\frac{R_{s r} i_{s \alpha}^{s} \lambda_{s r \beta}^{s}-\frac{d \lambda_{s r \alpha}^{s}}{d t} \lambda_{s r \beta}^{s}-R_{s r} i_{s \beta}^{s} \lambda_{s r \alpha}^{s}+\frac{d \lambda_{s r \beta}^{s}}{d t} \lambda_{s r \alpha}^{s}}{\lambda_{s r \alpha}^{s}{ }^{2}+\lambda_{s r \beta}^{s}{ }^{2}}$ 
This equation can be used as a speed estimator, provided that the flux linkage must be estimated from another equation, different from (7). In this case, one can use the voltage model as follows [9] [10]:

$$
\lambda_{s r}^{s}=\int_{-\infty}^{t}\left(u_{s}^{s}-R_{s} i_{s}^{s}\right) d t-L_{t} i_{s}^{s}
$$

As known, the digital implementation of a pure integral is not feasible due to the very well known drift problems. The problem is even more important since the speed estimation is based on an open-loop equation, so it must be coped with particular care. In this work, the solution presented in [11] has been used, enabling good performances at low speed. The only drawback is that the PI regulator, used to compensate the drift, must be tuned by a rule of thumb. The OLSE has been used in combination with a standard FOC drive, as depicted in Figure 2.

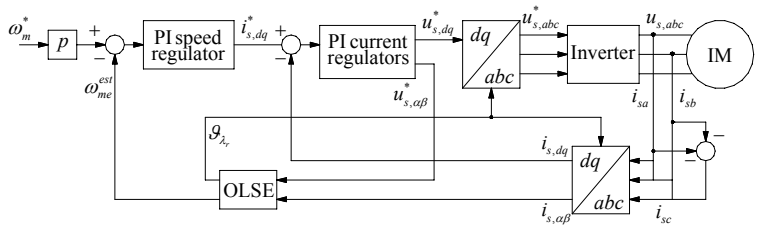

Figure 2. Sensorless OLSE block schematic

The OLSE is a computationally-light way of estimating the speed, which gives the drive the sensorless feature. The overall drive is not meant to be neither a closed loop estimator solution, like MRAS or Kalman filters, nor an open loop solution, like a $\mathrm{V}-\mathrm{Hz}$ algorithm.

\section{SENSITIVITY ANALYSIS}

Let $f(x, p)$ be a generic real function of $x$, with $p$ real positive parameter. The sensitivity function is conventionally defined as the ratio between $d f / f$, which is the relative variation of the function $f$, with respect to $d p / p$, which is the relative variation of $p$. Therefore, the sensitivity can be viewed as the total derivative of $f$ with respect to $p$, multiplied by $p / f$, which normalises the sensitivity in the selected working point:

$$
\tilde{S}_{f}^{p}(x, p)=\frac{d f / f}{d p / p}=\frac{d f}{d p} \frac{p}{f}
$$

The major drawback of (10) is its inherent singularity for $f=0$, due to the normalisation factor $p / f$.

This fact, which is obvious in principle, may alter a correct comparison of the sensitivities around $f=0$. In the present case $f$ is the electromechanical speed $\omega_{m e}$, and (10) wuld give imprecise results around zero. The problem can be tackled by using a slightly different sensitivity definition, in which the variations of $f$ and $p$ are related to their nominal values, instead of the actual ones:

$$
S_{f}^{p}(x, p)=\frac{d f}{d p} \frac{p_{N}}{f_{N}}
$$

So, a unitary sensitivity function indicates that a percentage error on the parameter $p$ would be reflected to an equivalent percentage error on $f$, compared to their respective nominal values. Consequently, the more the sensitivity function approaches zero, the less the function $f$ is influenced by an error on parameter $p$.

\section{A. V-Type drive sensitivity}

The sensitivity analysis for the V-type drive is not trivial, since the system is not based on a mathematical model for the speed estimation. Actually, the speed estimation is intrinsic and equal to the reference value imposed by the user. The consequence of a motor parameters mismatch has to be analysed considering the whole electrical and mechanical system.

For the sake of a more systematic and general analysis, the paper adopts the notation introduced in (6). If an error in the parameters vector $p$ occurs, it becomes:

$$
\frac{d(x+\Delta x)}{d t}=A(p+\Delta p, x+\Delta x)
$$

Expression (12) points out that wrong parameter values lead to a different state variables vector, and it also introduce an error in the speed estimation.

In principle, a sensitivity function can be obtained by taking the difference between (12) and (6), at steady-state. This is equivalent to drive the system in a steady-state condition with the right parameters, and suddenly perturb it with a step-like modification of one of the parameters of vector $p$. After a transient due to the error dynamics, the system reaches a new steady-state condition, presumably with a speed error, which is actually the sensitivity of the speed to the modified parameter.

As mentioned, the error dynamics can be obtained from the difference from (12) and (6) as follows:

$$
\begin{aligned}
\frac{d(x+\Delta x)}{d t}-\frac{d x}{d t} & =\frac{d \Delta x}{d t}=A(p+\Delta p, x+\Delta x)-A(p, x)= \\
& =\widetilde{A}(x, p, \Delta x, \Delta p)
\end{aligned}
$$

The system (13) is still non linear. Under the hypothesis of small parameter variations (and presumably small speed errors) around an operating point, all of the secondorder terms in (13) can be neglected, obtaining a linear system that approximates the error dynamics in the considered working point:

$$
\frac{d \Delta x}{d t}=\widetilde{A}(x, p, \Delta p) \Delta x
$$

At steady state, the system (14) can be solved in a closed form with respect to $\Delta x$. An approximation of the sensitivity function for each of the state variables is obtained by dividing (14) by the forced parameter variation $\Delta p$ (taking the limit for $\Delta p \rightarrow 0$ ) and normalising the result by the multiplication with $p / x$.

The sensitivity functions of the speed $\omega_{m e}$ with respect of each motor parameter have been obtained by forcing only one parameter variation at the time, while maintaining the others at their nominal value. In this way only scalar operations are involved. Anyway, it should be possible to obtain more complex vector solutions, in which the sensitivity functions could be analysed with 
respect to simultaneous variations of more than one motor parameter. The closed form calculation of the sensitivity functions required a relatively high computational effort. The obtained expressions have revealed to be very long and in some way not practical for a human insight. As an example, the next formula reports the sensitivity function of the electromechanical speed with respect to $R_{s r}$, which is the shortest among the four ones:

$$
\begin{aligned}
S_{\omega_{m e} R_{s r}}= & {\left[p ^ { 2 } i _ { Q } i _ { D } \left(\left(\lambda_{s r d} i_{Q} R_{s r}-\lambda_{s r d} \omega_{m e} L_{\varphi} i_{D}-R_{s r} i_{D} \lambda_{s r q}+\right.\right.\right.} \\
& \left.+i_{Q} R_{s r} L_{\varphi} i_{D}+\lambda_{s r d} \omega_{m e}^{r e f} L_{\varphi} i_{D}\right) \lambda_{s r d}^{r e f}+ \\
& +\omega_{m e} \lambda_{s r q}{ }^{2} L_{\varphi} i_{D}+i_{D}{ }^{2} R_{s r} L_{\varphi} \lambda_{s r q}+ \\
& \left.\left.-L_{\varphi} \lambda_{s r d} R_{s r} i_{D} i_{Q}+\omega_{m e} L_{\varphi} \lambda_{s r d}{ }^{2} i_{D}\right) R_{s r}\right] / \\
& {\left[\left(\lambda _ { s r d } ^ { r e f } \left(\left(-3 p^{2} \omega_{m e}^{r e f} i_{D}{ }^{2} L_{\varphi}+3 p^{2} i_{D}{ }^{2} \omega_{m e} L_{\varphi}+\right.\right.\right.\right.} \\
& \left.-3 p^{2} i_{Q} i_{D} R_{s r}\right) \lambda_{s r q}{ }^{2}+3 p^{2} i_{D} R_{s r} i_{Q} \lambda_{s r d}{ }^{2}+ \\
& -2 \omega_{m e}^{r e f} i_{D}{ }^{2} L_{\varphi} B_{m} R_{s r}+\left(3 p^{2} i_{Q}{ }^{2} R_{s r} \lambda_{s r d}+\right. \\
& -3 p^{2} i_{D} L_{\varphi} \omega_{m e} i_{Q} \lambda_{s r d}+3 p^{2} \omega_{m e}^{r e f} i_{D} L_{\varphi} \lambda_{s r d} i_{Q}+ \\
& \left.-3 p^{2} i_{D}{ }^{2} R_{s r} \lambda_{s r d}+3 p^{2} i_{D} L_{\varphi} R_{s r} i_{Q}{ }^{2}\right) \lambda_{s r q}+ \\
& \left.+2 i_{D}{ }^{2} B_{m} \omega_{m e} L_{\varphi} R_{s r}+3 p^{2} i_{D}{ }^{2} L_{\varphi} i_{Q} R_{s r} \lambda_{s r d}\right)+ \\
& +3 p^{2} i_{D}{ }^{2} R_{s r} \lambda_{s r q}{ }^{3}+2 \omega_{m e}^{r e f} i_{D}{ }^{3} L_{\varphi}{ }^{2} B_{m} R_{s r}+ \\
& +3 p^{2} \omega_{m e}^{r e f} i_{D}{ }^{3} L_{\varphi}{ }^{2} \lambda_{s r d}{ }^{2}+\left(6 p^{2} i_{D}{ }^{2} L_{\varphi} i_{Q} R_{s r}+\right. \\
& \left.-3 p^{2} i_{D} i_{Q} R_{s r} \lambda_{s r d}+3 p^{2} \omega_{m e}^{r e f} i_{D}{ }^{3} L_{\varphi}{ }^{2}\right) \lambda_{s r q}{ }^{2}+ \\
& +2 i_{D}{ }^{2} B_{m} i_{Q} L_{\varphi} R_{s r}{ }^{2}-2 i_{D} B_{m} R_{s r}{ }^{2} i_{Q} \lambda_{s r d}+ \\
& -2 i_{D}{ }^{2} B_{m} \omega_{m e} L_{\varphi} R_{s r} \lambda_{s r d}+\lambda_{s r q}\left(3 p^{2} i_{D}{ }^{2} R_{s r} \lambda_{s r d}{ }^{2}+\right. \\
& +3 p^{2} i_{D}{ }^{3} L_{\varphi} R_{s r} \lambda_{s r d}+2 i_{D}{ }^{2} B_{m} R_{s r}{ }^{2}+ \\
& \left.-3 p^{2} i_{D} L_{\varphi} i_{Q}{ }^{2} R_{s r} \lambda_{s r d}-2 i_{D} L_{\varphi} B_{m} \omega_{m e} R_{s r} i_{Q}\right)+ \\
& \left.\left.-3 p^{2} i_{D} i_{Q} R_{s r} \lambda_{s r d}{ }^{3}\right) \omega_{m e, N}\right] \\
&
\end{aligned}
$$

The expressions for the sensitivity functions $S^{R s}$ wme $S^{L t}{ }^{L m e}$ and $S^{L_{\varphi}}{ }_{\text {ome }}$, along with (15), have been used for a graphical comparison between the V-Type and the OLSE drive, as will be reported later on in the paper.

\section{B. OLSE drive sensitivity}

In case of the OLSE drive, calculation of the sensitivity functions is simpler, since the sensitivity formula (11) can be directly applied on (8) and (9). In this case, the obtained sensitivity functions refer to the estimated speed, that is $S_{\text {w.me. }}^{p}$. However, as far as the FOC drive is capable of following the estimated speed as the feedback signal, an error in the estimated speed will affect the actual speed with the opposite sign. Consequently, $S^{p}{ }_{\omega m e}$ is simply equal to $-S_{\hat{\text { wme }}}^{p}$.

A steady-state condition in which currents, voltages and flux linkages assume a sinusoidal profile is needed to obtain an algebraic solution of the sensitivity itself. As an example, $S_{\text {wme }}^{R S}$ results:

$$
S_{\hat{\omega}_{m e}}^{R_{s}}=\frac{R_{s} R_{s r} I_{s}^{2} \cos (2 \varphi)}{\omega_{s} \omega_{m e, N} \Lambda_{s r}{ }^{2}}
$$

where $I_{s}$ is the stator current magnitude, $\Lambda_{s r}$ is the rotor flux linkage magnitude referred to the stator, $\omega_{s}$ is the exciting frequency, $\omega_{m e, N}$ is the rated electromechanical speed, and $\varphi$ is the angle between stator current vector and rotor flux vector (torque angle). The other sensitivity functions, obtained with the same procedure, are the following [9]:

$$
\begin{gathered}
S_{\hat{\omega}_{m e}}^{R_{s r}}=-\frac{R_{s r} I_{s} \sin (\varphi)}{\omega_{m e, N} \Lambda_{s r}} \\
S_{\hat{\omega}_{m e}}^{L_{t}}=-\frac{R_{s r} L_{t} I_{s}{ }^{2} \sin (2 \varphi)}{\omega_{m e, N} \Lambda_{s r}{ }^{2}} \\
S_{\hat{\omega}_{m e}}^{L_{\varphi}}=0
\end{gathered}
$$

\section{V-Type and OLSE sensitivity comparison}

Figure 3 to 6 show the sensitivity function comparison between the V-Type drive and the OLSE drive, based on the expressions of Sec. IV-A and IV-B.

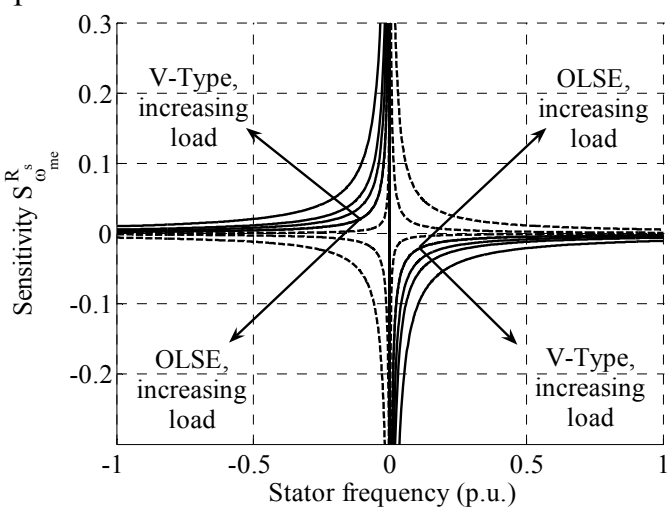

Figure 3. $S^{R s}{ }_{\text {ame }}$ as function of $\omega_{m e}$ and load torque.

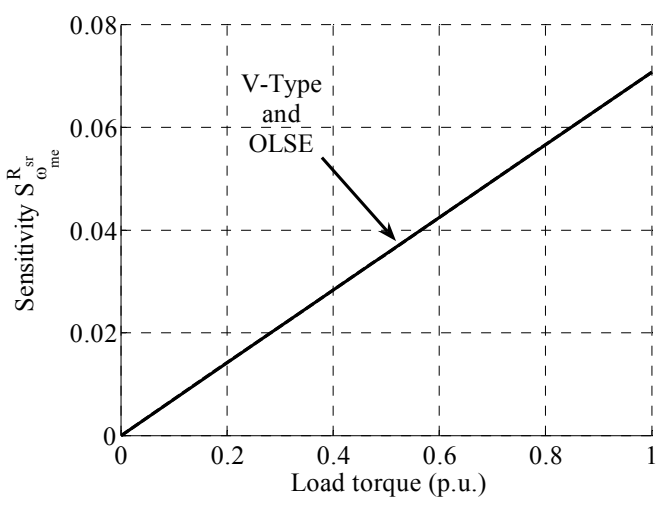

Figure 4. $S^{R s r}{ }_{\text {ome }}$ as function of load torque.

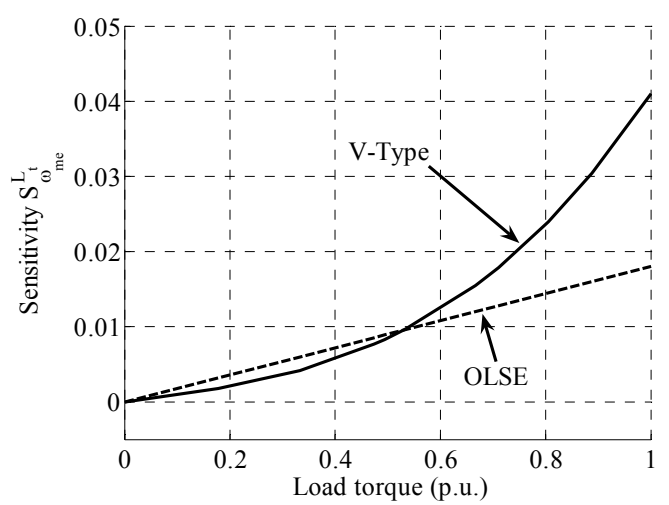

Figure 5. $S^{L t}{ }_{\text {ome }}$ as function of load torque. 


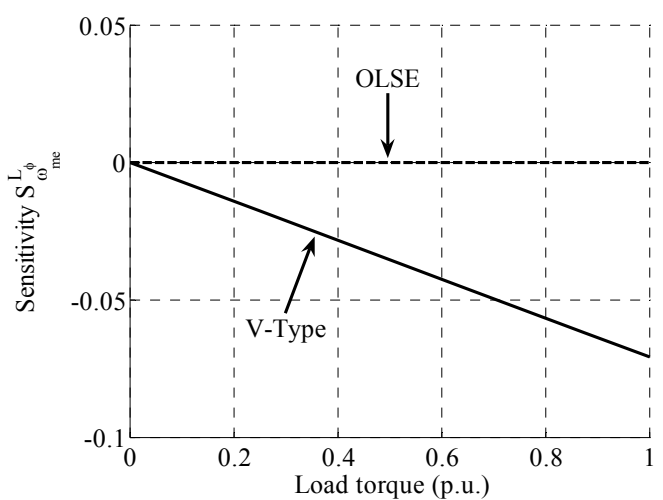

Figure 6. $S^{L \varphi}$ wme as function of load torque.

As can be seen, the OLSE sensitivity function $S^{R s}$ behaves quite differently with respect of that of the VType drive. They coincide at no load, as highlighted by the same starting point for the arrows in Figure 3. Interestingly, the OLSE sensitivity can be very low in case of medium loads (see the numerator of (16), which is zero for $\varphi=\pi / 4)$, while for the V-Type drive it is always increasing along with the load torque. However, both sensitivities are infinite as speed approaches zero, revealing the yet unsolved problem of sensorless algorithms.

Only the sensitivity to Rs variation is function of the speed itself, and that is why such dependence appears in Figure 3 and not in the others.

Sensitivities $S^{R s r}$ ome are practically the same for both algorithms. Sensitivities $S^{L t}{ }_{\text {wme }}$ reveal that, in this case, the V-Type drive is only slightly better at light loads, while its robustness is worse at full load (quadratic crescent profile against the OLSE linear crescent profile). Finally, sensitivities $S^{L \varphi}$ cme show that the OLSE drive is unaffected by a $L_{\varphi}$ variation, while the V-Type drive is still sensitive because of the $\omega_{s l}$ calculation shown in Figure 1.

\section{EXPERIMENTAL RESULTS}

Parameter sensitivities of the two drives have been experimentally compared to the numerical analysis of Sect. IV. A parameter variation can be easily obtained by forcing via software a change of the correct motor parameters used by the control algorithm. Such variation is introduced on-the-fly by means of a flag within the control algorithm, causing a transient due to the sudden change of the parameters themselves. These transients are not meaningful since motor parameters slowly vary in a real motor, so they have been removed in the following figures, and only the steady-state values have been left.

Different experiments have been performed to validate the analytical expressions. In a first batch, the drive was rotating at $300 \mathrm{rpm}$. Four parameter variations have been forced in the control algorithms, recording the steady state speed at no load and at rated load: $-40 \%$ of $R_{s}^{*},-40 \%$ of $R_{s r}{ }_{s r}$ (both to recall temperature effects), $+20 \%$ of $L^{*}{ }_{t}$ and $+20 \%$ of $L_{\varphi}^{*}$ (both to recall saturation effects).

Figures 7 to 14 show the experimental steady-state speeds of both OLSE and V-Type algorithms compared to the reference speed, at no load and at rated load as well. Table I summarizes the experimental results, comparing them to the analytical calculations performed with the expressions of Sec. IV. It can be noted that speed variations follow the theoretical analysis of Sec. IV for both algorithms at any load condition. The only measurement which shows a large discrepancy to the analytical calculation refers to the $R_{s}$ variation at full load, for the OLSE drive. This can be explained by considering the steep profile of the sensitivity curve of Figure 3 at low speeds, where a little variation in the stator frequency causes a rather large speed variation.

A second batch of measurements has been performed for both algorithms at no load and different speeds, forcing a $-40 \%$ variation of the $R_{s}$ used by the control algorithm. Table II shows the experimental steady-state speed errors compared to the analytical results. As can be seen, prediction seems to be closer to the experiments as the speed increases. This result can be explained again considering both the steepness of the sensitivity curves, which in turn causes the effect of hardware non-ideality (dead times, measurement noise, and so forth) to be amplified.

It is worth to note that the speed error signs are coherent with the analysis of Sec. IV. In particular, the better behaviour of the OLSE drive at full load is experimentally validated after the theoretical profile shown in Figure 3.

TABLE I.

STEADY-STATE SPEED ERROR AT NO LOAD AND RATED LOAD (AT 300 RPM)

\begin{tabular}{c|c|c|c|c|c}
\multicolumn{2}{c|}{} & \multicolumn{2}{c|}{ No load } & \multicolumn{2}{c}{ Rated load } \\
\cline { 3 - 7 } \multicolumn{2}{c|}{} & $\begin{array}{c}\text { Analyt. } \\
(\mathrm{rpm})\end{array}$ & $\begin{array}{c}\text { Experim. } \\
(\mathrm{rpm})\end{array}$ & $\begin{array}{c}\text { Analyt. } \\
(\mathrm{rpm})\end{array}$ & $\begin{array}{c}\text { Experim. } \\
(\mathrm{rpm})\end{array}$ \\
\hline \multirow{4}{*}{$\Delta R_{s}$} & OLSE & 27 & 20 & -41.3 & -6 \\
\cline { 2 - 6 } & V-Type & 27 & 20 & 71.6 & 75 \\
\hline \multirow{4}{*}{$\Delta R_{s r}$} & OLSE & 0 & 0 & -84.8 & -80 \\
\cline { 2 - 6 } & V-Type & 0 & 0 & -84.8 & -80 \\
\hline \multirow{3}{*}{$\Delta L_{t}$} & OLSE & 0 & 0 & 10.8 & 10 \\
\cline { 2 - 6 } & V-Type & 0 & 0 & 24.6 & 20 \\
\hline \multirow{3}{*}{$\Delta L_{\varphi}$} & OLSE & 0 & 5 & 0 & -8 \\
\cline { 2 - 6 } & V-Type & 0 & 0 & -42.4 & -34
\end{tabular}

TABLE II.

STEADY-STATE SPEED ERROR DUE TO $R_{S}$ VARIATION AT NO LOAD (AS FUNCTION OF SPEED).

\begin{tabular}{c|c|c|c} 
& $\begin{array}{c}\text { Analyt. } \\
(\mathrm{rpm})\end{array}$ & $\begin{array}{c}\text { OLSE } \\
\text { Exp. (rpm) }\end{array}$ & $\begin{array}{c}\text { V-Type } \\
\text { Exp. (rpm) }\end{array}$ \\
\hline $100 \mathrm{rpm}$ & 82.9 & 40 & 43 \\
\hline $200 \mathrm{rpm}$ & 41.5 & 27 & 28 \\
\hline $300 \mathrm{rpm}$ & 27.6 & 20 & 20 \\
\hline $400 \mathrm{rpm}$ & 20.7 & 15 & 16 \\
\hline $500 \mathrm{rpm}$ & 16.6 & 12.5 & 12.5 \\
\hline $1000 \mathrm{rpm}$ & 8 & 4.5 & 6
\end{tabular}




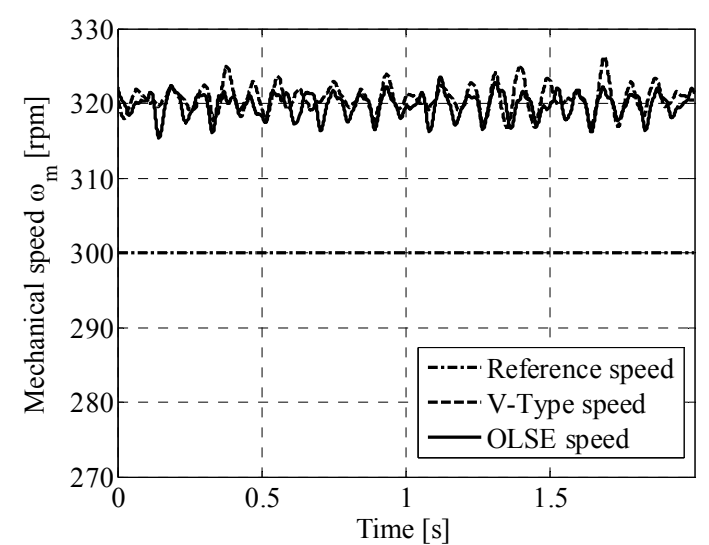

Figure 7. Experimental results: no load, $-40 \%$ variation of $R_{s}$.

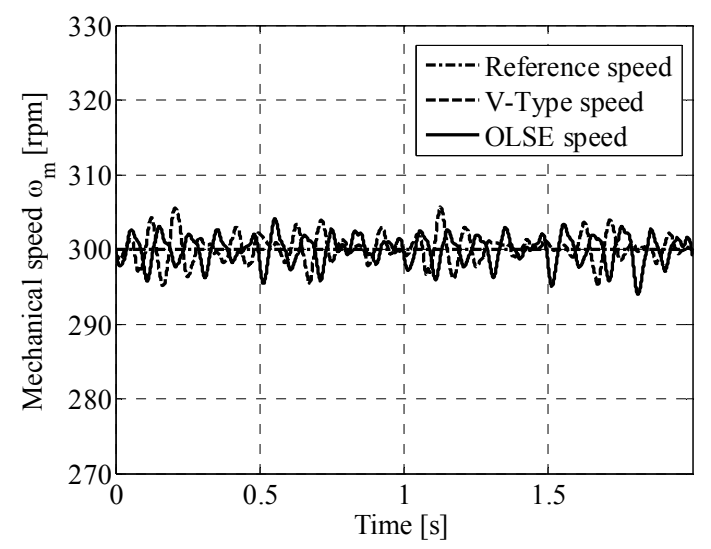

Figure 8. Experimental results: no load, $-40 \%$ variation of $R_{s}$.

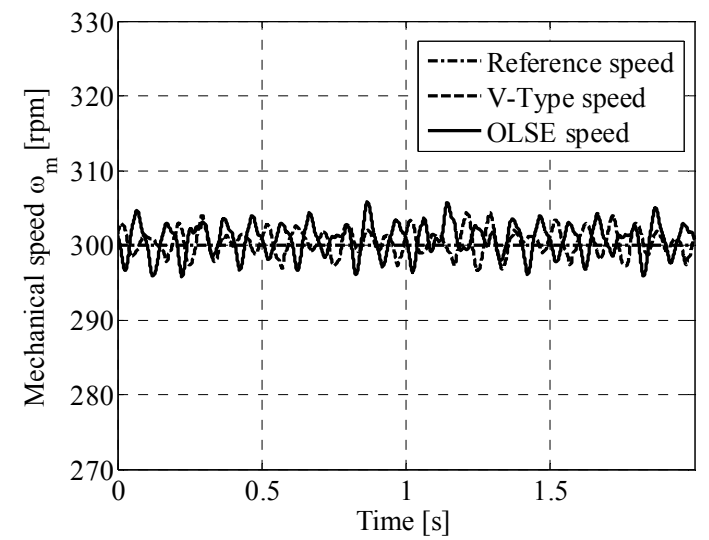

Figure 9. Experimental results: no load, $+20 \%$ variation of $L_{t}$.

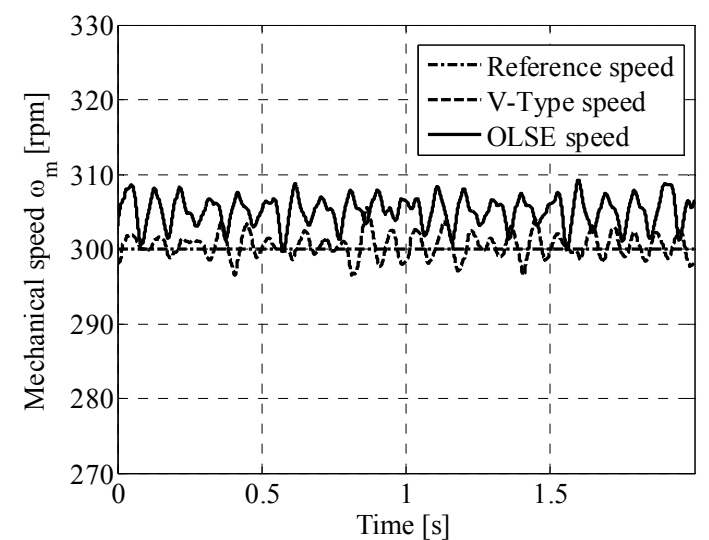

Figure 10. Experimental results: no load, $+20 \%$ variation of $L_{\varphi \text { r }}$

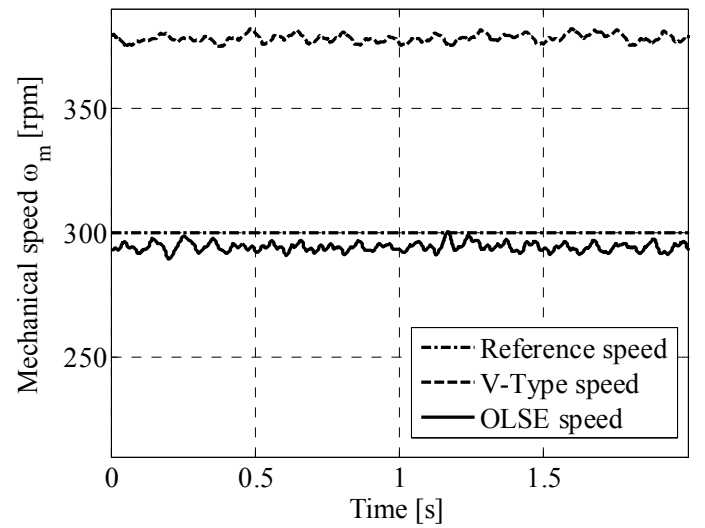

Figure 11. Experimental results: rated load, $-40 \%$ variation of $R_{s}$.

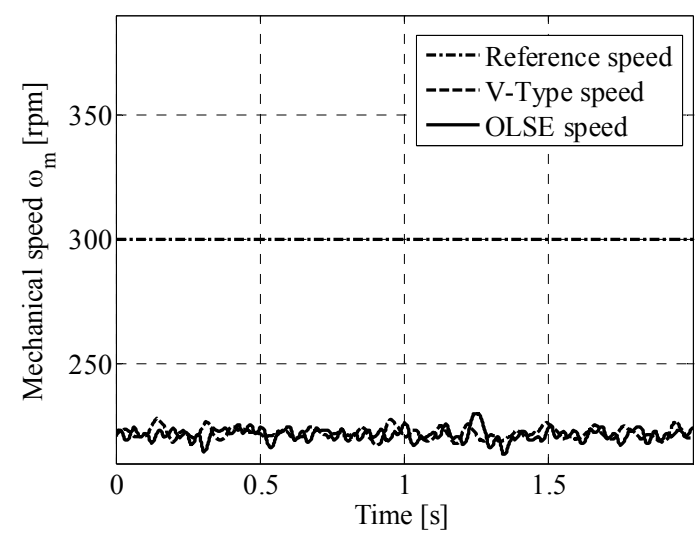

Figure 12. Experimental results: rated load, $-40 \%$ variation of $R_{s r}$

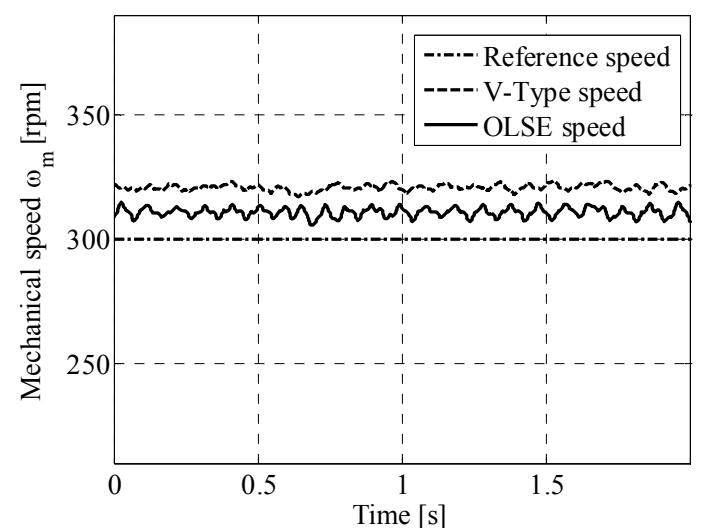

Figure 13. Experimental results: rated load, $+20 \%$ variation of $L_{t}$.

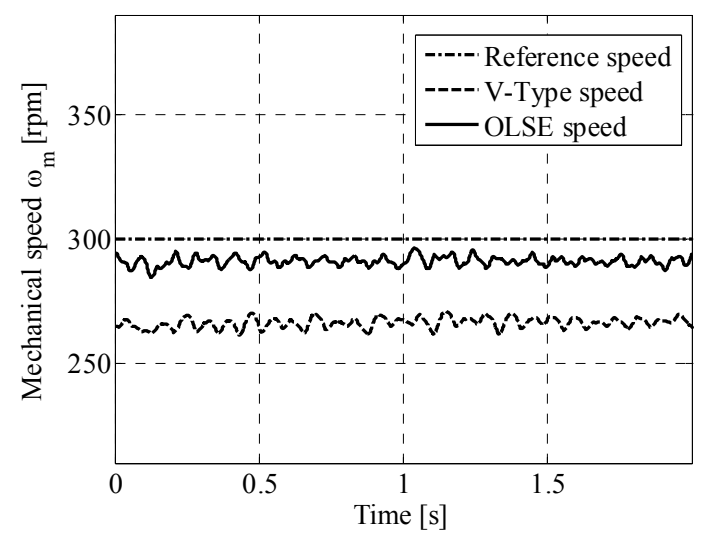

Figure 14. Experimental results: rated load, $+20 \%$ variation of $L_{\varphi}$ 


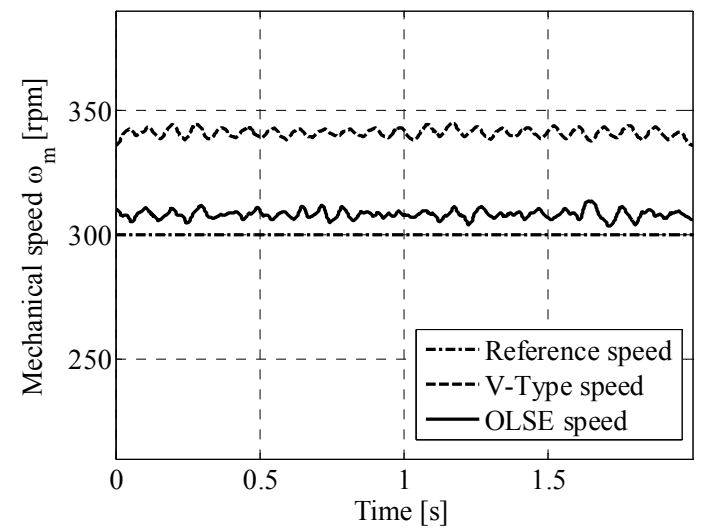

Figure $15.50 \%$ of rated load, $-40 \%$ variation of $R_{s}$.

TABLE III.

INDUCTION MOTOR PARAMETERS.

\begin{tabular}{l|c} 
Nominal speed $\omega_{N}$ & $3000 \mathrm{rpm}$ \\
\hline Nominal torque $\tau_{N}$ & $3.8 \mathrm{Nm}$ \\
\hline Nominal current $I$ & $3.2 \mathrm{Arms}$ \\
\hline Pole pairs p & 2 \\
\hline Stator resistance $R_{s}$ & $8.04 \Omega$ \\
\hline Rotor resistance referred to the stator $R_{s r}$ & $4.96 \Omega$ \\
\hline Transient inductance $L_{t}$ & $26.8 \mathrm{mH}$ \\
\hline Magnetising inductance $L \varphi$ & $210.3 \mathrm{mH}$
\end{tabular}

\section{CONCLUSIONS}

The paper copes with the comparison of two different low-cost sensorless control schemes for induction motors. The analysis has focused on the sensitivity of the two alternatives to single parameter variations. The comparison has been based on analytical sensitivity functions, verified by experimental tests. The paper faces successfully both practical and methodological facets. In conclusion, the drive based on the open loop speed estimator demonstrates to be slightly better than the VType drive in all the sensitivity functions, with the exception of $S^{R s}{ }^{R m}$, which is practically the same for the two schemes. As a side results, the availability of the analytical sensitivity functions may also represent an interesting tool for parameters estimation and tracking. This issue will be one of the next research topics.

\section{REFERENCES}

[1] P. Vas, Sensorless Vector and Direct Torque Control, Oxford University Press ed., UK, 1998.

[2] T. Du, P. Vas, and F. Stronach, "Design and Application of Extended Observers for Joint State and Parameter Estimation in High-Performance AC Drives," IEE Proc. Electr. Pow. Appl., vol. 142, no. 2, pp. 71-78, Mar. 1995.

[3] Y.-R. Kim, S.-K. Sul, and M.-H. Park, "Speed Sensorless Vector Control of Induction Motor Using Extended Kalman Filter," IEEE Trans. Ind. Appl., vol. 30, no. 5, pp. 1225-1233, Sep./Oct. 1994.

[4] M. Rashed and A. F. Stronach, "A Stable Back-EMF MRASbased Sensorless Low-Speed Induction Motor Drive Insensitive to Stator Resistance Variation," IEE Proc.-Electr. Power Appl., vol. 151, no. 6, pp. 685-693, Nov. 2004.

[5] S. Jin, Z. Wei, and H. Zhenyi, "One Novel Scalar Control Scheme for Induction Machine," in Proc. of 30th Annual Conf. of IEEE Industrial Electronics Society (IECON 2004), vol. 1, Nov. 2004, pp. 347-352.

[6] C. C. Wang and C. H. Fang, "Sensorless Scalar Controlled Induction Motor Drives with Modified Flux Observer," IEEE Power Engineering Review, vol. 22, no. 8, pp. 61-68, Aug. 2002, ISSN: 0272-1724.

[7] S. Bolognani, L. Tubiana, and M. Zigliotto, "V-Type Sensoreless Field Oriented Control of Induction Motors for Low Cost Applications," in Proc. of (EPE-PEMC 2004), vol. CDROM.

[8] S. Bolognani, A. Faggion, L. Peretti, and M. Zigliotto, "Sensorless V-Type Vector-Controlled IM Drive with Inherent FluxWeakening Capability," in Proc. of the 4th IET Conference on Power Electronics, Machines and Drives (PEMD 2008), Apr. 2-4, 2008, pp. 465-469.

[9] S. Bolognani, L. Peretti, and M. Zigliotto, "A Novel Open-Loop Speed Estimation Technique for Low-Cost IM Drives," in Proc. of the ACEMP \& Electromotion 2007 Joint Conference, Bodrum, Turkey, Sep. 2007, pp. 754-759.

[10] J. Holtz and J. Quan, "Sensorless Vector Control of Induction Motors at Very Low Speed Using a Nonlinear Inverter Model and Parameter Identification,” IEEE Trans. Ind. Appl., vol. 38, no. 4, pp. 1087-1095, Jul./Aug. 2002.

[11] J. Hu and B. Wu, "New Integration Algorithms for Estimating Motor Flux over a Wide Speed Range," IEEE Trans. Power Electron., vol. 13, no. 5, pp. 969-977, Sep. 1998. 